\title{
Low Flow Voltage Free Interface for Capillary Electrophoresis and Mass Spectrometry Driven by Vibrating Sharp-edge Spray Ionization
}

\author{
Courtney J. Kristoff, Chong Li, Peng Li, and Lisa A. Holland* \\ C. Eugene Bennett Department of Chemistry, West Virginia University, \\ Morgantown, West Virginia 26506, United States
}

*Corresponding author contact information: Lisa.Holland@mail.wvu.edu

\begin{abstract}
This material includes data that demonstrates the dependence of ionization on the acoustic source, plate counts and migration times obtained with an ammonium acetate background electrolyte at $\mathrm{pH} 6.5$, and mass spectra of peptides present in soybean trypsin inhibitor sample.

Author contributions are as follows: CJK: Methodology (CE capillary fabrication, CE-UV analyses), Validation (all CE-MS), Formal Analysis, Writing-Reviewing and Editing, Original Draft preparation, Visualization. CL: Methodology (VSSI probe fabrication/alignment), Validation (CE-MS of $\beta$-blockers/peptides). PL: Conceptualization, Methodology, Supervision, Project Administration, Funding Acquisition. LAH: Conceptualization, Methodology, Validation, Writing-Reviewing and Editing, Visualization, Supervision, Project Administration, Funding Acquisition.
\end{abstract}

\section{TABLE OF CONTENTS}

Information

Page

Figure S1. Effect of acoustic source on ionization

S-1

Table S1. Separation efficiency per meter at pH 6.5 (VSSI-MS vs CE-UV)

S-2

Table S2. Migration time at $\mathrm{pH} 6.5$ (VSSI-MS vs CE-UV)

S-3

Figure S2. Mass spectra of peptides in protein sample (pH 6.5)

S-4 


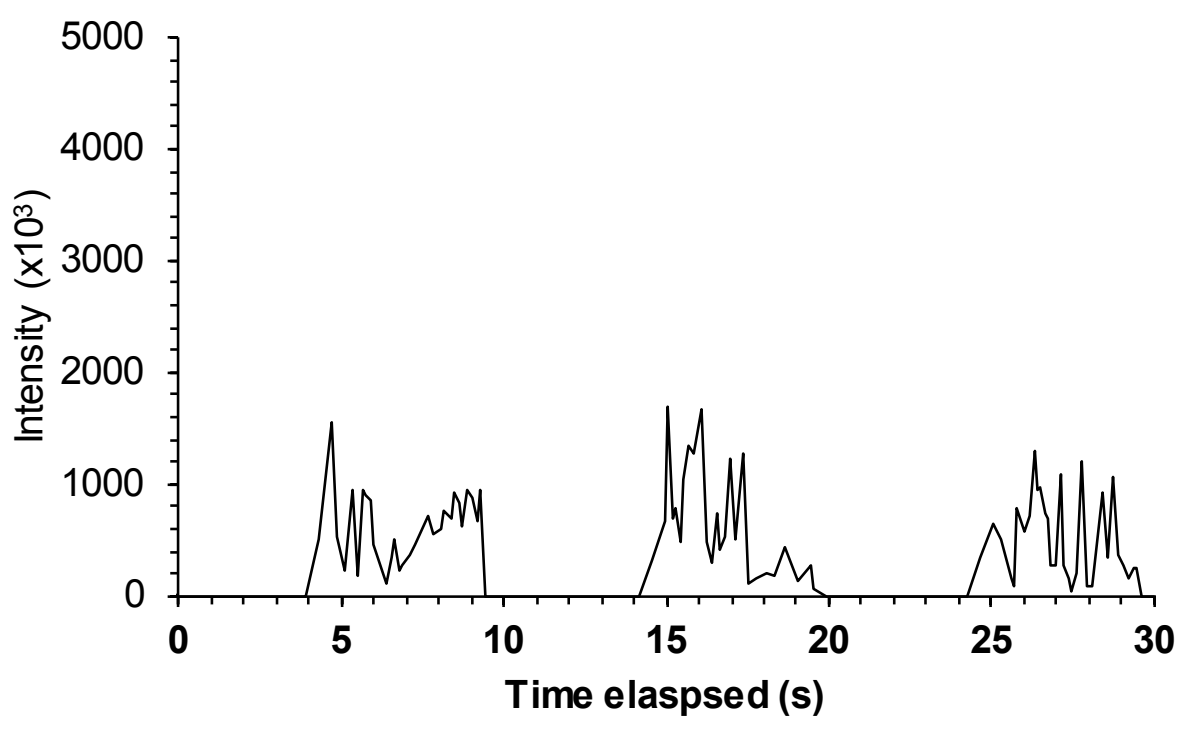

Figure S1. Demonstration that ionization starts and stops with acoustics. A voltage of $10 \mathrm{kV}$ was applied to the CE capillary during the experiment. The acoustics were pulsed on and off in 5-sec increments starting with an off period of $0-5$ sec elapsed. The extracted ion electropherogram depicts ionization intensity for pindolol which is plotted as the extracted base peak of 249.3 with a mass tolerance of $500 \mathrm{mmu}$ 


\section{Table S1. Separation efficiency per meter at pH 6.5 (VSSI-MS vs CE-UV)}

\begin{tabular}{|c|c|c|}
\hline & CE-MS $(n=3)^{b}$ & CE-UV $(n=3)$ \\
\hline $\mathrm{pH} 6.5$ & Plates (RSD) & Plates (RSD) $^{\mathrm{d}}$ \\
\hline Pindolol & $70,000(40)$ & $80,000(10)$ \\
\hline Acebutolol & $80,000(20)$ & $80,000(20)$ \\
\hline Somatostatin & $11,000(90)$ & $10,000(60)$ \\
\hline Oxytocin & $30,000(40)$ & $40,000(40)$ \\
\hline Ubiquitin & $10,000(50)$ & $17,500(5)$ \\
\hline Trypsin inhibitor & $30,000(40)$ & $17,000(2)$ \\
\hline
\end{tabular}

Table S2. Migration time at pH 6.5 (VSSI-MS vs CE-UV)

\begin{tabular}{lcc}
\hline & CE-MS $(\boldsymbol{n}=3)$ & CE-UV $(\boldsymbol{n}=3)$ \\
\cline { 2 - 3 } pH 6.5 & Time (RSD) & Time (RSD) \\
Pindolol & $3.59(0.5)$ & $3.84(1)$ \\
Acebutolol & $3.85(0.7)$ & $4.06(1)$ \\
Somatostatin & $4.7(10)$ & $4.6(3)$ \\
Oxytocin & $4.9(10)$ & $5.06(0.7)$ \\
Ubiquitin & $7.5(2)$ & $5.60(1)$ \\
Trypsin inhibitor & $11.0(1)$ & $7.61(0.8)$
\end{tabular}

${ }^{\mathrm{a}}$ Distance to detector is $40 \mathrm{~cm}$.

${ }^{b}$ Values for peak width were obtained with 2 significant figures.

'Values for peak width were obtained with 4 significant figures.

${ }^{\mathrm{d} C a l c u l a t e d}$ by determining the width at base $(\mathrm{w})$ as $\mathrm{N}=16 \mathrm{t}^{2} / \mathrm{w}^{2}$ 

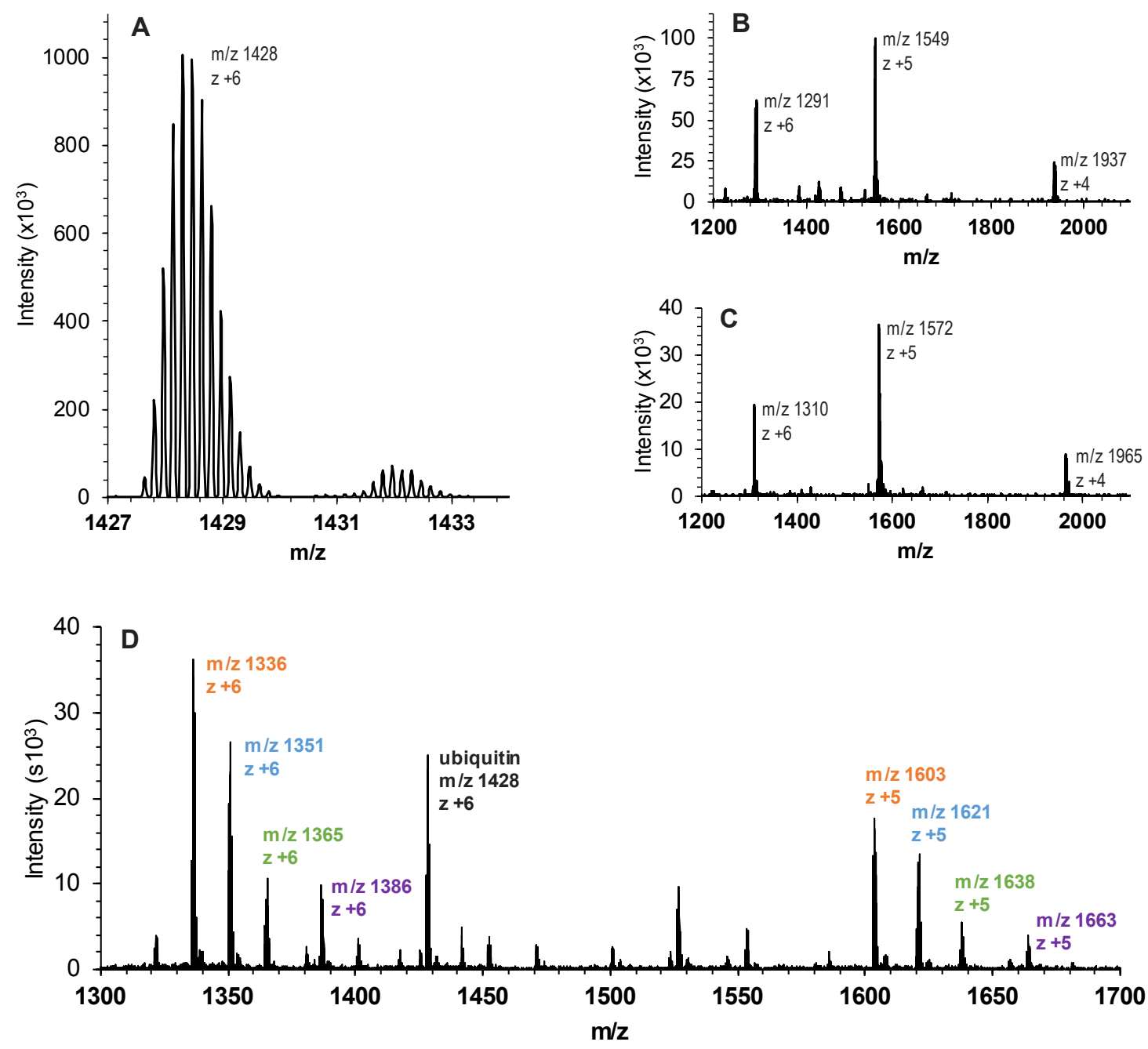

Figure S2. (A) Mass spectra containing the dominant charge state of ubiquitin. (B) Mass spectrum of the peptides in the trypsin inhibitor with masses of 7746, (C) 7860, (D) 8016 in orange, 8106 in blue, 8190 in green, and 8316 in purple. Separation conditions are listed in Fig. 4. 